\title{
Psicología
}

Artículo original

\section{Habilidades relacionadas con la comprensión lectora en lectores iniciales que crecen en contextos de pobreza}

\section{Skills related to reading comprehension in early readers growing up in poverty contexts}

\author{
Marina Ferroni ${ }^{1 凶} \underline{\mathrm{ORCID}}$
}

${ }^{1}$ Doctora en Psicología. Instituto de Lingüística, Facultad de Filosofía y Letras (UBA). Consejo Nacional de Investigaciones Científicas y Técnicas (CONICET, Argentina).

Fecha correspondencia:

Recibido: septiembre 6 de 2019.

Aceptado: junio 4 de 2021.

\section{Forma de citar:}

Ferroni, M. (2021). Habilidades relacionadas con la comprensión lectora en lectores iniciales que crecen en contextos de pobreza. Rev. CES Psico, 14(3), 1-18. https://dx.doi.org/10.21615/ cesp. 5188

\section{Open access}

C Derecho de autor Licencia creative commons Ética de publicaciones Revisión por pares Gestión por Open Journal System DOI: 10.21615/cesp.5188

ISSNe: 2011-3080

Publica con nosotros

\section{Resumen}

El presente trabajo se propuso analizar la contribución de la comprensión de lenguaje oral y la velocidad y la precisión en la lectura de palabras en la comprensión lectora en lectores iniciales del español que crecen en contextos de pobreza urbana. Para ello, se evaluó a 31 niños de 3er grado que crecían en barrios socialmente vulnerables de la provincia de Buenos Aires, Argentina, mediante pruebas de comprensión lectora, precisión y velocidad lectora, vocabulario y procesamiento morfosintáctico. Los resultados de la muestra total indican que la medida de comprensión lectora se asoció a las medidas de vocabulario, procesamiento de lenguaje oral, y velocidad y precisión en la lectura. En contraposición a resultados obtenidos en otras lenguas de ortografía transparente, el análisis de una regresión indicó que la comprensión lectora estuvo mayormente explicada por la medida de precisión y no de velocidad en la lectura de palabras. A fin de enriquecer el análisis de los datos, a partir de los resultados en la prueba de comprensión de textos se conformaron dos grupos de niños con diferente nivel de comprensión lectora: un grupo de niños con un nivel de comprensión lectora cercano a la media obtenida ( 21 sujetos) y un grupo de niños cuyo desempeño se ubicó en un desvío por debajo de la media en la medida de 
comprensión (10 sujetos). Los resultados de una prueba de comparación de medias mostraron que entre grupos de niños con diferente nivel de comprensión lectora las habilidades que se diferenciaron fueron las relacionadas al procesamiento morfosintáctico.

Palabras clave: comprensión lectora, nivel lector, habilidades lectoras, dificultades lectoras, lenguas transparentes, pobreza.

\section{Abstract}

The present study aims to analyze the contribution of oral language and reading comprehension skills in early readers of Spanish who grow up in poverty context in Buenos Aires, Argentina. For this, reading comprehension, reading accuracy, reading fluency, vocabulary and morphosyntax processing were tested in 31 3rd graders. Full sample data analysis show that reading comprehension measures was associated with vocabulary, oral language processing, reading speed and accuracy. In contrast to results obtained in other languages with transparent orthographies, regression analysis shows that reading comprehension was mainly explained by reading accuracy and not by reading speed. In order to improve the data analysis, two groups of children with different levels of reading comprehension were selected: one group (21 children) whose level of comprehension was near to the average obtained and one group of children (10 subjects) with lower reading comprehension level. The results of a mean comparison test showed that in groups of children with different levels of reading comprehension, the skills that differed were those related to morphosyntactic processing.

Keywords: reading comprehension, reading level, reading skills, reading difficulties, transparent languages, poverty.

\section{Introducción}

El objetivo del presente estudio es analizar el impacto de la comprensión de lenguaje oral y la velocidad y la precisión en la lectura en la comprensión lectora en lectores iniciales del español que crecen en contextos de pobreza urbana.

La comprensión de textos, es decir, la construcción de significado a partir de un texto escrito por parte del lector es el objetivo último del aprendizaje de la lectura (Gentaz, SprengerCharolles, \& Theurel, 2015). En la vida escolar de los niños, la adecuada comprensión de textos escritos resulta fundamental, dado que constituye el principal mecanismo mediante el cual los niños incorporan nuevos conocimientos (Defior, 2015). 
La comprensión lectora ha sido definida como una habilidad compleja en la cual intervienen innumerables procesos como el conocimiento previo, la generación de inferencias, el nivel de memoria operativa y otros mecanismos cognitivos de los lectores que posibilitan la conformación de una representación mental del texto escrito (Nation, 2005; Ouellette \& Beers, 2010; Perfetti, Landi, \& Oakhill, 2005; Van den Broek, 1994; Van den Broek, Espin, McMaster, \& Helder, 2017). Esta perspectiva de la comprensión lectora ha sido desarrollada hace ya muchos años en el campo de la psicología experimental (Hoover \& Gough, 1990).

Por otra parte, la Simple View of Reading (Visión Simple de la Lectura en español, de ahora en más VSL) (Gough \& Tunmer, 1986; Hoover \& Gough, 1990) plantea que muchas de las habilidades que intervienen en la construcción del significado de los textos escritos son, en realidad, habilidades generales de compresión lingüística ligadas al nivel de vocabulario y al nivel de conocimiento de reglas morfológicas y sintácticas que intervienen en la compresión del lenguaje tanto oral como escrito (Gough \& Tunmer, 1986; Hoover \& Gough, 1990).

La evidencia empírica que sustenta las ideas de la VSL se relaciona con el hecho de que el nivel de compresión de lenguaje de los sujetos resulta estable a pesar de que la información lingüística sea presentada en muy diversos soportes (Kendeou, Van den Broek, White, \& Lynch, 2009). En este sentido, existe un gran número de estudios que analizaron la comprensión lingüística en distintos formatos (de manera escrita, de manera oral o en medios audiovisuales) y sugirieron que las habilidades de compresión de lenguaje oral y el nivel de vocabulario de los sujetos impactaban en el nivel de compresión de las personas más allá del soporte utilizado (Oakhill \& Cain, 2012; Spencer, Quinn, \& Wagner, 2014). Estos datos sugieren la existencia de habilidades lingüísticas que permiten comprender el lenguaje más allá de la forma en que este sea presentado y que, a estas habilidades de compresión lingüística, se le suman habilidades específicas de acceso a la información en cada tipo de soporte en particular.

La VSL postula, que las habilidades para comprender textos escritos dependen básicamente de dos habilidades generales consideradas independientes: las habilidades de compresión de lenguaje oral y la decodificación de palabras escritas (Gough \& Tunmer, 1986; Hoover \& Gough, 1990).

Existe un importante número de trabajos que aportan evidencia a favor de esta línea de análisis de la comprensión lectora (Caravolas et al., 2019; Ferroni \& Jaichenco, 2020; Hulme, 2019; Kim, 2017; Martins \& Capellini, 2018; Snow, 2018). 
En líneas generales, los trabajos realizados en este marco reportan una relación compleja entre las habilidades que contribuyen a la comprensión de textos escritos y la existencia de factores que pueden mediar dicha relación: la edad o grado escolar de los niños y el nivel de transparencia de la ortografía en cuestión (Gentaz et al., 2015; Gentaz, Sprenger-Charolles, Theurel, \& Colé, 2013).

Estudios longitudinales realizados en inglés señalaron que la medida de lectura de palabras fue la variable que tuvo más incidencia (por sobre la medida de comprensión de lenguaje oral) en la compresión lectora en los momentos iniciales del aprendizaje de la lectura de los niños. En estos trabajos también se observó que el nivel de lectura de palabras siguió teniendo un importante impacto en la medida de comprensión lectora luego de 3 a 5 años de instrucción (Droop \& Verhoeven, 2003; Florit \& Cain, 2011).

Ahora bien, el inglés es una lengua de ortografía opaca con un gran nivel de inconsistencias entre los sonidos de la lengua y sus representaciones escritas y en la cual los sujetos tienden a necesitar períodos un tanto prolongados para desarrollar fluidez en la lectura (Wimmer \& Schurz, 2010). Por el contrario, el español es una lengua de ortografía transparente con un alto grado de consistencia en la relación fonología-ortografía y en la cual los niños alcanzan rápidamente altos niveles de precisión lectora (Landerl \& Wimmer, 2008; Signorini \& Borzone de Manrique, 2003). No resultaría sorprendente entonces que, en estos sistemas alfabéticos, el impacto de las habilidades lectoras sobre la comprensión sea más acotado.

En este sentido, en estudios realizados en ortografías más transparentes que el inglés se ha observado que la velocidad lectora tiene mayor incidencia que la comprensión oral en la comprensión lectora, en el primer año de instrucción formal en la lectura (Lepola, Lynch, Kiuru, Laakkonen, \& Niemi, 2016; Tobia \& Bonifacci, 2015).

Sin embargo, estos estudios también señalan que, la relativa contribución de los dos componentes principales implicados en la comprensión lectora, se encontrarían afectados por un factor adicional, esto es, si la habilidad de decodificación de palabras es medida en términos de precisión o de velocidad en la lectura (Florit, Roch, Dicataldo, \& Levorato, 2020).

En efecto, la evidencia obtenida en diferentes investigaciones sugiere que la precisión y la velocidad en la lectura de palabras, si bien se encuentran estrechamente ligadas, son construcciones separables en ortografías transparentes (Protopapas et al., 2013). La precisión en la lectura se alcanza a partir de un correcto proceso de recodificación fonológica, es decir, 
una vez los lectores iniciales desarrollan un alto nivel de conocimiento de las correspondencias entre letras y sonidos, y cierto nivel de conciencia fonológica que les permite unir los sonidos para pronunciar la palabra (Aro \& Lyytinen, 2016). Por otra parte, la velocidad que permite el reconocimiento automático de las palabras se desarrolla a partir de que los sujetos almacenan representaciones ortográficas que especifican las letras que componen las palabras y la ubicación de los grafemas dentro de ellas (Perfetti, 1992).

En esta línea, un metaanálisis realizado con lectores iniciales que leían en ortografías más transparentes que el inglés (Florit \& Cain, 2011) señaló que el nivel de comprensión oral tuvo una influencia mayor que la precisión lectora en la comprensión lectora. Ahora bien, cuando se midió el nivel de incidencia de la comprensión oral y la velocidad lectora en la comprensión oral se observó que la velocidad lectora contribuyó en mayor medida que la comprensión de lenguaje oral a los procesos de comprensión de textos de lectores iniciales.

Existen, sin embargo, otros estudios que han informado hallazgos mixtos con respecto a la contribución relativa de la precisión y la velocidad lectora en la comprensión lectora, por lo tanto, la contribución de cada habilidad es motivo de debate (Roch \& Levorato, 2009; Tobia \& Bonifacci, 2015).

Específicamente en español, diversos estudios realizados señalaron que la velocidad lectora impactó de modo directo en las medidas de comprensión de textos narrativos y expositivos (De Mier, Amado, \& Benítez, 2015; De Mier, Borzone, \& Cupani, 2012). Sin embargo, estos estudios han sido realizados en poblaciones sin carencias materiales graves, razón por la cual resultaría interesante indagar sobre las habilidades implicadas en la comprensión lectora en niños de nivel socio económico bajo.

En efecto, existe en Argentina, un $48 \%$ de niños que crecen en contextos de pobreza. En las afueras de Buenos Aires, lugar donde fue realizado el presente estudio, las condiciones de vida de estos niños y sus familias son muy duras dado que un importante porcentaje de estos niños habitan viviendas superpobladas (25\%), con falta de agua potable $(21 \%)$ o con falta de redes de gas natural (83\%) (Diuk, Barreyro, Ferroni, Mena, \& Serrano, 2019; Tuñón, 2018).

Respecto de los factores educativos, numerosas investigaciones que comparan el desempeño en la lectura de niños de diferentes sectores socioeconómicos han señalado que los que crecen en entornos menos favorecidos suelen presentar un menor nivel de desarrollo lector que los niños de otros sectores sociales (Gentaz et al., 2013; 2015; Urquijo, Coni, \& Fernandes, 2015). 
Estas diferencias se explicaron a partir de factores experienciales como la calidad de las propuestas educativas a las que acceden los niños de sectores desfavorecidos (Ferroni, Barreyro, Mena, \& Diuk, 2019; Diuk, Ferroni, Mena, \& Barreyro, 2017) o a otros factores, como la percepción sobre estos niños que tienen las instituciones educativas a las que asisten (idea del déficit o déficit thinking en inglés, Valencia, 2010).

Por otra parte, a pesar de que existen numerosos estudios intergrupales que comparan el desarrollo del proceso de alfabetización en niños de diferente nivel socioeconómico, los estudios intragrupales en niños que crecen en contextos de pobreza ha recibido mucha menos atención (García Coll, 2015; Diuk et al., 2017). Dado que se sugiere la existencia de altos niveles de variabilidad intragrupal en el desempeño lector en niños que crecen en estos contextos (Diuk et al., 2019), resultaría de sumo interés analizar el impacto de cada una de las habilidades relacionadas con la comprensión de textos escritos en niños de estos sectores.

Por todo lo expuesto hasta aquí, el presente trabajo se propone analizar la incidencia de la comprensión oral, la precisión y la velocidad en la lectura en la comprensión de textos en una ortografía transparente como el español y en lectores iniciales que crecen en contextos de pobreza.

\section{Método}

La presente investigación se enmarca en un enfoque metodológico cuantitativo, de diseño no experimental y trasversal, con un alcance correlacional-causal (Hernández Sampieri, Fernández Collado, \& Baptista Lucio, 2014).

\section{Participantes}

Fueron incluidos en el estudio 31 niños (14 varones y 17 mujeres) de $3 e r$ grado (edad, $M=8,8$ años; D.E. $=6.1$ ) de una escuela parroquial de un barrio de bajos recursos del conurbano bonaerense (Argentina).

Respecto de las escuelas parroquiales en las cuales se evaluó a los niños, las mismas se asientan en un gran número de barrios de bajos recursos del conurbano bonaerense y dependen de los obispados de cada lugar, los cuales administran la gestión de cada escuela. En dichas instituciones, los fondos destinados a los salarios de los docentes son otorgados por el estado provincial, hecho que permite que las familias de los niños que concurren a estas instituciones deban abonar una baja cuota mensual. Estas escuelas constituyen espacios de cohesión social para los niños y las familias en situación de vulnerabilidad social (Tuñón \& Di Paolo, 2018). 
Respecto de la medida de nivel socioeconómico (NSE) de los sujetos, como es común en las sociedades latinoamericanas, las personas que viven en contextos socialmente vulnerados sufren segregación residencial (entre otras), hecho que genera la conformación de grandes áreas de viviendas precarias con bajos niveles de servicios básicos (Sabatini, 2003) que tienden a ser socialmente homogéneas (Groisman \& Suárez, 2009). En efecto, ha sido señalada en numerosas ocasiones la existencia de una asociación entre el lugar de residencia y el nivel socioeconómico de los sujetos (Diuk et al., 2019; Hackman \& Farah, 2009; Hanscombe et al., 2012), razón por la cual el lugar de residencia de los sujetos fue tomado en el presente estudio como indicador socioeconómico per se.

Como información adicional sobre el NSE de los niños incluidos en el estudio, se les pidió a los directivos del colegio información (que permanecería confidencial) sobre la ocupación de los adultos responsables de los niños. El estado ocupacional de los padres se clasificó siguiendo la escala ocupacional de Sautú (1992), que organiza las ocupaciones de acuerdo con nueve categorías. El uso de esta escala está relacionado con el hecho de que fue diseñada para incluir incluso trabajos típicos de la economía argentina.

La totalidad de los adultos que informaron empleo tenían ocupaciones clasificadas en las tres categorías inferiores de la escala (trabajadores informales de la construcción, empleadas de servicio doméstico, empleados informales). No se solicitó información sobre ingresos dado que consultar sobre este punto no constituye una práctica habitual en Argentina en ningún nivel socioeconómico. Por otra parte, específicamente en el caso de comunidades de bajo NSE, dada la informalidad de los empleos a los cuales acceden los adultos, los ingresos poseen un alto índice de variación diaria hecho que dificulta a las personas poder calcular las ganancias anuales o incluso mensuales (Diuk et al., 2019). Con anterioridad al comienzo de las sesiones de evaluación, las familias de todos los niños firmaron un consentimiento informado expresando su conformidad para que estos participen en el proyecto de investigación. En dicho consentimiento, se le aclaraba a las familias que la información obtenida sería confidencial de acuerdo con lo que establece la Ley № 25.326 de Protección de datos personales (Ley de Habeas Data) y que los datos personales de los niños y sus familias no serían divulgados públicamente, y serían utilizados únicamente los resultados de las pruebas a fin de contribuir con la investigación.

Los procedimientos aplicados en la toma de datos respetaron en todo momento los principios de la Convención Internacional de los Derechos del Niño y las legislaciones nacionales (argentinas) e internacionales referidas a los derechos de los niños. 
Asimismo, los niños participantes manifestaron su asentimiento en forma verbal. En todo el transcurso de las sesiones de trabajo, la evaluadora estuvo atenta a que no se produjera ningún tipo de malestar que justificara la exclusión de algún niño del estudio.

Con el fin de realizar un análisis adicional de los datos obtenidos, a partir de los resultados en la prueba de comprensión de textos (Cuetos Vega, 2014) se formaron dos grupos de niños con diferente nivel de comprensión: un grupo de niños con un nivel de comprensión lectora cercano a la media obtenida (21 sujetos) y un grupo de niños cuyo desempeño se ubicó en un desvío por debajo de la media en la medida de comprensión.

\section{Materiales}

Comprensión lectora: con el fin de medir el nivel de comprensión lectora de los niños, se administró el sub-test de comprensión de textos de la batería Prolec $R$ (Cuetos Vega, 2014). En esta prueba los niños deben leer un texto narrativo de aproximadamente 90 palabras de manera silente. Luego de la lectura, los niños deben responder de forma escrita cuatro preguntas: dos preguntas sobre información literal del texto leído y dos preguntas sobre información que los niños debían inferir. Se asignaba un punto a cada respuesta correcta.

Lectura de palabras: con el fin de medir la precisión y la velocidad en la lectura de palabras, se administró el test Prolec $R$ (Cuetos Vega, 2014). La prueba consta de 40 ítems de diferente longitud y nivel de complejidad silábica, que los participantes deben leer en voz alta. Se asignó un punto a cada palabra correctamente decodificada y se tomó el tiempo de lectura para calcular la cantidad de palabras por minuto que lograban leer correctamente los niños.

Habilidades de comprensión oral Vocabulario: Se administró la prueba de Vocabulario en Imágenes del test Peabody (Dunn, 1986). En esta prueba, la evaluadora iba mostrando a los niños diferentes láminas, cada una con cuatro ilustraciones (objetos manufacturados, animales, acciones humanas, plantas, etc.). En cada lámina se les iba diciendo una palabra a los niños y éstos debían seleccionar, en cada la lámina, la ilustración que correspondía a la palabra. Se asignó un punto a cada respuesta correcta. En los resultados se exponen los puntajes brutos.

Procesamiento morfosintáctico: se administró el sub-test "Estructura de oraciones" de la batería CELF 4 (Semel, Wiig, \& Secord, 2006) que permite analizar el procesamiento y la comprensión oral de diferentes tipos de oraciones. El sub-test consta de 31 oraciones que son leídas a los niños mientras observan cuatro imágenes diferentes. Luego de escuchar la frase que lee el experimentador, el niño debe señalar la imagen que corresponde a la frase leída. 


\section{Procedimiento}

La prueba de comprensión lectora fue administrada grupalmente en la escuela y en el aula en la cual los niños realizan sus tareas escolares diariamente. Las demás pruebas fueron administradas en dos sesiones individuales de evaluación de aproximadamente 20 minutos cada una. La toma de datos se llevó a cabo entre los meses de octubre y noviembre, es decir, al finalizar el año escolar. Todas las pruebas fueron administradas por la autora de este trabajo.

\section{Resultados}

En primer lugar, se analizaron las distribuciones de las puntuaciones obtenidas en las tareas administradas. Este análisis permitió observar que ninguna de las medidas se alejó de la distribución normal asintótica.

Asimismo, se calcularon los estadísticos descriptivos de todas las variables incluidas en el estudio. Los resultados se muestran en la tabla 1.

Tabla 1. Estadísticos descriptivos de las medidas obtenidas en la muestra total.

\begin{tabular}{lcc} 
& M & D.E. \\
Comprensión lectora & 72.58 & 25.29 \\
\hline Velocidad lectora (pal. x & 33.58 & 9.67 \\
\hline Precisión lectora & 89.38 & 18.19 \\
Vocabulario & 39.32 & 10.92 \\
\hline Procesamiento & 83.76 & 9.87
\end{tabular}

Por otra parte, con el fin de explorar qué habilidades se relacionaron con la medida de comprensión lectora, se calcularon las correlaciones de todas las medidas evaluadas. Los resultados se muestran en la tabla 2 . 
Tabla 2. Correlaciones de todas las habilidades evaluadas en la muestra total.

1- Comprensión lectora

2- Velocidad lectora

3- Precisión lectora

4-Vocabulario

5- Procesamiento morfosintáctico

${ }^{*} \mathrm{p}<.05 ; * \mathrm{p}<.01 ; * *$

$\begin{array}{ccccc}\mathbf{1} & \mathbf{2} & \mathbf{3} & \mathbf{4} & \mathbf{5} \\ 1 & .408^{*} & .579^{* *} & .403^{*} & .389^{*} \\ .408^{*} & 1 & .490^{* *} & .337 & .316 \\ .579^{* *} & .490^{* *} & 1 & .316 & .442^{*} \\ .403^{*} & .337 & .316 & 1 & .205 \\ .389^{*} & .316 & .442^{*} & .205 & 1\end{array}$

El análisis de las correlaciones señaló la existencia de correlaciones positivas, significativas y moderadas entre las habilidades de comprensión lectora y las demás habilidades evaluadas.

Además, con el fin de analizar la incidencia de las habilidades de procesamiento del lenguaje oral y las habilidades de lectura (precisión y velocidad) en la comprensión lectora en la muestra total, se realizaron una serie de regresiones por pasos sucesivos con la medida de comprensión lectora como variable dependiente y las demás medidas evaluadas como variables independientes. Los resultados se muestran en la tabla 3.

Tabla 3. Porcentaje de varianza de los predictores de la medida de comprensión lectora.

Predictores
Precisión en la lectura
$\begin{array}{ll} & 56.9 * * \\ \text { Total, R2 } & 33.6 \\ * \mathrm{p}<.05 ;{ }^{* *} \mathrm{p}<.01 ; * * * \mathrm{p}<.001 . & \end{array}$

Los resultados de las regresiones sugieren que, en la muestra total de sujetos, la medida de comprensión en la lectura fue explicada mayormente por el nivel de precisión en la lectura de palabras.

Por último, con el fin de enriquecer el análisis de los datos obtenidos, se formaron dos grupos de niños con diferente nivel de comprensión lectora: un grupo de niños con un nivel de comprensión lectora cercano a la media obtenida (21 sujetos) y un grupo de niños cuyo desempeño se ubicó en un desvío por debajo de la media en la medida de comprensión (10 sujetos). Dado que los grupos quedaron conformados por un pequeño número de niños, se realizaron una serie de pruebas no paramétricas de contrastes de medias ( $U$ de Wilcoxon). El 
análisis señaló la existencia de diferencias entre los grupos en la prueba de procesamiento morfosintáctico $(z=-2.03, p=.040)$. Este dato sugiere la existencia de un pequeño grupo de niños que presentó mayores dificultades para comprender textos y que no tenía diferencias en el nivel de lectura de palabras en la comparación con sus pares, pero sí en procesamiento morfosintáctico.

\section{Discusión}

El presente estudio se propuso analizar la contribución de la comprensión de lenguaje oral y la velocidad y la precisión en la lectura de palabas en la comprensión lectora en lectores iniciales del español que crecen en contextos de pobreza urbana. Para ello, se evaluó a 31 niños de 3er grado que crecían en barrios socialmente vulnerados de la provincia de Buenos Aires mediante pruebas de comprensión lectora, comprensión oral (vocabulario receptivo y procesamiento morfosintáctico) y habilidades de lectura.

En primer lugar, el análisis de los datos arroja evidencia en relación con los postulados de la VSL. En efecto, la medida de comprensión lectora se relacionó con las medidas de reconocimiento de palabras y con las medidas de vocabulario y de procesamiento morfosintáctico.

En este sentido, los datos concuerdan con investigaciones que plantean la importancia del vocabulario para la comprensión de textos. La amplitud y diversidad léxica posibilitaría conectar las ideas del texto y relacionar dichas ideas con la información almacenada en la memoria (Cromley, Snyder-Hogan, \& Luciw-Dubas, 2014). Asimismo, los resultados obtenidos están en línea con investigaciones que sugieren que, para poder comprender lenguaje oral o textos escritos, los niños deben ser capaces de procesar sintácticamente la información que se presenta. Se entiende que el procesamiento sintáctico permite establecer las relaciones entre los constituyentes oracionales y proyectar esta información para obtener el significado de las oraciones (Brimo, Lund, \& Sapp, 2018).

Por otra parte, los resultados de las regresiones señalaron que la medida de comprensión lectora fue mayormente explicada por la medida de precisión en la lectura de palabras.

En efecto, los análisis realizados en el marco de la VSL señalan que, para acceder al significado de los textos, a las habilidades de comprensión de lenguaje oral se le suman habilidades específicas de acceso a la información en cada tipo de soporte en particular, en este caso, el reconocimiento de palabras escritas (Spencer et al., 2014). 
En este sentido, estudios longitudinales realizados en diferentes lenguas han sostenido que existe un cambio diacrónico en la contribución que realizan la habilidad de lectura de palabras y la comprensión oral a la comprensión lectora durante el desarrollo de los niños (Kim, 2017; Martins \& Capellini, 2018; Snow, 2018). En la primera fase de la instrucción formal de alfabetización (es decir, dentro del primer y tercer año de instrucción), la fuerza de la predicción de la comprensión lectora a partir del reconocimiento de palabras es mayor que la de la comprensión oral.

Estudios realizados en lenguas más transparentes que el inglés han señalado que la medida de reconocimiento de palabras podría tener un impacto menor en lectores iniciales, dada la transparencia del sistema ortográfico (Caravolas et al., 2019). Sin embargo, estudios realizados en italiano, una lengua casi tan transparente como el español (Dehaene, 2015), Ilamaron la atención sobre un factor adicional que podría estar incidiendo en la contribución de la medida de lectura de palabras en la comprensión de textos: la forma en que se mide la lectura de palabras (atendiendo a la velocidad en la lectura o a la precisión) (Florit et al., 2020; Florit \& Cain, 2011). En estos estudios se observó que la medida de comprensión oral realizaba una contribución mayor sobre la comprensión lectora cuando se tomaba en cuenta la medida de precisión en la lectura de palabras. Sin embargo, cuando se tomaba como medida de lectura la medida de velocidad en la lectura esta era la habilidad que realizaba una contribución mayor a la comprensión de textos escritos aún en lectores iniciales de una ortografía transparente.

Los datos arrojados en el presente estudio tuvieron en cuenta tanto la medida de precisión como de velocidad lectora y, en contraposición a los estudios italianos mencionados, en lectores iniciales argentinos que crecen en contextos de pobreza fue la medida de precisión lectora la que contribuyó de mayor manera a la medida de comprensión de textos.

Vale la pena aclarar que los niños evaluados presentaron un desempeño calificado como de "lectores muy lentos" en la comparación con los baremos de la prueba utilizada para evaluar lectura, lo que podría indicar que todavía utilizan mayormente un proceso de recodificación fonológica para acceder a la lectura y no un procesamiento de acceso al léxico.

El bajo nivel de velocidad lectora podría ser explicado por el nivel socioeconómico de los sujetos ya que numerosas investigaciones que comparan el desempeño en la lectura de niños de diferentes sectores socioeconómicos han señalado que los que crecen en entornos menos favorecidos suelen presentar un menor nivel de desarrollo lector (Gentaz et al., 2013; 2015; Urquijo et al., 2015). 
A pesar del bajo nivel de velocidad lectora presentado por los niños participantes del presente estudio, el nivel de precisión en la lectura les permitió comprender en cierta medida textos narrativos simples, hecho que sugiere que, en una lengua de alto nivel de transparencia ortográfica, la precisión en la lectura resulta fundamental para el acceso al significado de los textos en lectores iniciales. Futuros estudios deberán abordar la dicotomía precisión/velocidad en la lectura y su relación con la comprensión de textos más extensos y complejos que los utilizados en el presente estudio.

Un interesante resultado adicional fue obtenido cuando se dividió a la muestra total en pequeños grupos según la medida de comprensión lectora obtenida. Este análisis, señaló la existencia de un pequeño grupo de niños que presentó mayores dificultades para comprender textos y que no tenía diferencias en el nivel de lectura de palabras en la comparación con sus pares, pero sí en procesamiento morfosintáctico. En este sentido, se ha señalado que existen niños que experimentan dificultades en la comprensión de textos escritos a pesar de tener habilidades de lectura de palabras apropiadas para su edad (Catts, Herrera, Nielsen, \& Bridges, 2015; Oakhill, Cain, \& Elbro, 2019). A pesar de que se podría asumir, a partir de la comparación de los resultados con los baremos de la prueba de lectura, que ninguno de los niños participantes posee un nivel lector acorde a su edad escolar, los resultados obtenidos en el presente estudio sugieren la existencia de un subgrupo de niños con problemas específicos de comprensión lectora que parecen explicarse, en principio, por un bajo nivel de procesamiento de lenguaje oral.

En conclusión, a diferencia de estudios llevados a cabo en otras lenguas de ortografía transparente (italiano, finés, entre otros), el presente estudio parece indicar que en lectores iniciales que crecen en contextos de pobreza, la habilidad que mayormente incide en la comprensión lectora es la precisión en el reconocimiento de palabras y que un nivel adecuado de recodificación fonológica les permite a los niños alcanzar, al menos, un nivel aceptable de comprensión de textos narrativos simples. Estos resultados concuerdan con otros realizados específicamente en español (De Mier, Amado, \& Benítez, 2015; De Mier, Borzone, \& Cupani, 2012), en los cuales se observó que en niños de escolaridad más avanzada que los del presente estudio fueron tanto las medidas de velocidad como de precisión en la lectura de palabras las que incidieron de manera directa en la medida de comprensión textual.

Por otra parte, los resultados obtenidos indican que existe un subgrupo de niños con un bajo nivel de comprensión de textos que poseen dificultades en el procesamiento morfosintáctico. 
Sin embargo, una limitación del presente estudio es el pequeño tamaño de la muestra por lo que estudios futuros deberán observar si estos resultados se replican con una muestra mayor.

Por último, los resultados obtenidos en el presente estudio permiten plantear importantes implicancias educativas. En efecto, los datos obtenidos sugieren que resulta necesario, sobre todo en niños de nivel socio económico bajo, los cuales suelen transitar menos experiencias extraescolares de alfabetización que niños de otros sectores sociales, intervenciones sistemáticas que, por un lado, permitan desarrollar mayores niveles de lectura para que sea posible acceder a la construcción de significados a partir de textos de mayor complejidad que los utilizados en el presente estudio. Por otra parte, los datos obtenidos sobre los niños que experimentaron dificultades para comprender textos señalan la importancia de llevar a cabo intervenciones educativas que permitan promover un desarrollo lingüístico integral y que impliquen, tempranamente, el tratamiento de lenguaje oral.

\section{Referencias}

Aro, M., \& Lyytinen, H. (2016). Training Reading Skills in Finnish: From Reading Acquisition to Fluency and Comprehension. In A. Khateby I. Bar-Kochva (Eds.), Reading Fluency: Current Insights from Neurocognitive Research and Intervention Studies (pp. 125-140). Literacy Studies: Springer International Publishing. https://doi.org/10.1007/978-3-319-30478-6 8 Brimo, D., Lund, E., \& Sapp, A. (2018). Syntax and reading comprehension: a meta-analysis of different spoken-syntax assessments. International Journal of Languaje \& Communications Disorders, 53, 431-445. https://doi.org/10.1111/1460-6984.12362

Caravolas, M., Lervag, A., Mikulajová, M., Defior, S., Málková, G., \& Hulme, Ch. (2019). A CrossLinguistic, Longitudinal Study of the Foundations of Decoding and Reading Comprehension Ability. Scientific Studies of Reading, 23(5), 386-402. https://doi.org/10.1080/10888438.2019.1580284

Catts, H., Herrera, S., Nielsen, D., \& Bridges, M. (2015). Early Prediction of Reading Comprehension within the Simple View Framework. Reading and Writing, 28, 1407-1425. https://doi.org/10.1007/s11145-015-9576-x

Cromley, J.G., Snyder-Hogan, L.E., \& Luciw-Dubas, Y.A. (2014). Cognitive activities in complex science text and diagrams. Contemporary Educational Psychology, 35, 59-74. doi: https://doi.org/10.1016/j.cedpsych.2009.10.002

Cuetos Vega, F. (2014). PROLEC-R: Batería de evaluación de los procesos lectores, revisada (5a ed.). Madrid: TEA.

Defior, S. (2015). Cómo mejorar la lectura. Mente y cerebro, 70, 16-23.

Dehaene, S. (2015). Aprender a leer. De las ciencias cognitivas al aula. Buenos Aires: Siglo XXI. 
De Mier, M. V., Amado, B., \& Benítez, M. E. (2015). Dificultades en la comprensión de textos expositivos en niños de los primeros grados de la escuela primaria. Psykhe (Santiago), 24(2), 1-13. http://dx.doi.org/10.7764/psykhe.24.2.708

De Mier, M. V., Borzone, A. M., \& Cupani, M. (2012). La fluidez lectora en los primeros grados: relación entre habilidades de decodificación, características textuales y comprensión. Un estudio piloto con niños hablantes de español. Neuropsicologia Latinoamericana, 4(1), 1833.

Diuk, B., Barreyro, J. P., Ferroni, M., Mena, M., \& Serrano, F. (2019). Reading Difficulties in LowSES Children: A Study of Cognitive Profiles. Journal of Cognition and Development, 20, 7595. https://doi.org/10.1080/15248372.2018.1545656

Diuk, B., Ferroni, M., Mena, M., \& Barreyro, J. (2017). Respuesta a la intervención y escritura en niños de grupos sociales vulnerados. Páginas de Educación, 10(2), 96-110. https://doi.org/10.22235/pe.v10i2.1426

Droop, M., \& Verhoeven, L. (2003). Language proficiency and reading ability in first-and secondlanguage learners, Reading Research Quarterly, 38(1), 78-103. https://doi.org/10.1598/RRQ.38.1.4

Dunn, L.M. (1986). Test de vocabulario en imágenes Peabody. Madrid: MEPSA.

Florit, E., \& Cain, K. (2011). The simple view of reading: is it valid for different types of alphabetic orthographies? Educational Psychology 576. https://doi.org/10.1007/s10648-011-9175-6

Florit, E., Roch, M., Dicataldo, R., \& Levorato, M.C. (2020). The Simple View of Reading in Italian beginner readers: Converging evidence and open debates on the role of the main components. Learning and Individual Differences. https://doi.org/10.1016/j.lindif.2020.101961.

Ferroni, M., Barreyro, J. P., Mena, M., \& Diuk, B. (2019). Perfiles cognitivos de niños de nivel socioeconómico bajo con dificultades en la velocidad lectora: análisis de los resultados de una intervención, Interdisciplinaria, 36, 1, 273-288.

Ferroni, M., \& Jaichenco, V. (2020). Comprensión lectora en contextos de pobreza: un análisis desde la Visión Simple de la Lectura. Lenguaje, 48(2), 225-240. https://doi.org/10.25100/lenguaje.v48i2.8610

Garcia Coll, C. (2015). Editorial: Continuity and change in child development. Child Development, 86, 7-9. https://doi.org/10.1111/cdev.2015.86.issue-1

Gentaz, E., Sprenger-Charolles, L., Theurel, A., \& Colé, P. (2013). Reading comprehension in a large cohort of French first graders from low socio-economic status families: A 7-month longitudinal study. Plos One, 8(11), e786082.

Gentaz, E., Sprenger-Charolles, L., \& Theurel, A. (2015). Differences in the predictors of reading comprehension in first graders from low socio-economic status families with either good or poor decoding skills, Plos One, 10(3), 0119581. https://doi.org/10.1371/journal.pone.0119581 
Gough, P. B., \& Tunmer, W. E. (1986). Decoding, reading, and reading disability. Remedial and Special Education, 7, 6-10. https://doi.org/10.1177/074193258600700104

Groisman, F., \& Suárez, L. (2009). Residential segregation in Greater Buenos Aires. In B. Roberts y R. Wilson (Eds.), Urban segregation and governance in the Americas (pp. 39-64). New York, NY: Palgrave-Macmillan.

Hackman, D. A., \& Farah, M. J. (2009). Socioeconomic status and the developing brain. Trends in Cognitive Sciences, 13(2), 65-73. https://doi.org/10.1016/i.tics.2008.11.003

Hanscombe, K. B., Trzaskowski, M., Haworth, C. M., Davis, O. S., Dale, P. S., \& Plomin, R. (2012). Socioeconomic status (SES) and children's intelligence (IQ): In a UK representative sample SES moderates the environmental, not genetic, effect on IQ. PLoS One, 7(2), e30320. https://doi.org/10.1371/journal.pone.0030320

Sampieri, R. H., Collado, C. F., Lucio, P. B., Valencia, S. M., \& Torres, C. P. M. (1998). Metodología de la investigación. México, DF: McGraw-Hill.

Hoover, W., \& Gough, P. (1990). The Simple View of Reading. Reading and Writing, 2, 127-160. Kendeou, P., Van den Broek, P., White, M. J., \& Lynch, J. S. (2009). Predicting reading comprehension in early elementary school: The independent contributions of oral language and decoding skills.Journal of Educational Psychology, 101, 765778. https://doi.org/10.1037/a0015956

Kim, Y., \& Wagner, R. (2015). Text (oral) reading fluency as a construct in reading development: An investigation of its mediating role for children from grades 1 to 4, Scientific Studies of Reading, 19, 224-242. https://doi.org/10.1080/10888438.2015.1007375

Landerl, K., \& Wimmer, H. (2008). Development of word reading fluency and spelling in a consistent orthography: an 8-year follow-up. Journal of Educational Psychology, 100(1), 150-161. https://doi.org/10.1037/0022-0663.100.1.150

Lepola, J., Lynch, J., Kiuru, N., Laakkonen, E., \& Niemi, P. (2016). Early oral language comprehension, task orientation, and foundational reading skills as predictors of grade 3 reading comprehension. Reading Research Quarterly, 51(4), 373-390. https://doi.org/10.1002/rrq.145

Martins, M., \& Cappellini, S. (2018). Relation between oral reading fluency and reading comprehension. CoDAS, 31(1), e20170244. https://doi.org/10.1590/2317$1782 / 20182018244$

Nation, K. (2005). Children's reading comprehension difficulties. In M. J. Snowling, C. Hulme, \& M. Seidenberg (Eds.), The science of reading: A handbook (pp. 248-265). Oxford: Blackwell.

Oakhill, J. V., \& Cain, K. (2012). The precursors of reading ability in young readers: Evidence from a four-year longitudinal study. Scientific studies of reading, 16(2), 91-121.

Oakhill, J. V., Cain, K., \& Elbro, C. (2019). Reading Comprehension and Reading Comprehension Difficulties. In D. A. Kilpatrick, R. M. Joshi, \& R. K. Wagner (Eds.), Reading Development and Difficulties: Bridging the Gap Between Research and Practice (pp. 83-115). Cham, Switzerland: Springer. https://doi.org/10.1007/978-3-030- 26550-2 5 
Ouellette G, Beers A. (2010). A not-so-simple view of reading: how oral vocabulary and visualword recognition complicate the story. Reading and Writing 23,189-208.

Perfetti, C.A. (1992). The representation problem in reading acquisition. En P.B. Gough, L.C. Ehri \& R. Treiman (Eds.), Reading acquisition (pp. 145-174). Hillsdale, NJ: Lawrence Erlbaum.

Perfetti, C. A., Landi, N., \& Oakhill, J. (2005). The acquisition of reading comprehension skill. In M. J. Snowling y C. Hulme (Eds.), The science of reading: A handbook (pp. 227-247). Oxford: Blackwell Publishing.

Protopapas, A., Mouzaki, A., Sideridis, G. D., Kotsolakou, A., \& Simos, P. G. (2013). The role of vocabulary in the context of the simple view of reading. Reading \& Writing Quarterly, 168202. https://doi.org/10.1080/10573569.2013.758569

Roch, M., \& Levorato, M. C. (2009). Simple view of reading in Down's syndrome: The role of listening comprehension and reading skills. International Journal of Language \& Communication Disorders, 44(2), 206-223. https://doi.org/10.1080/13682820802012061

Sabatini, F. (2003). La segregación social del espacio en las ciudades de América Latina. Chile: Banco Interamericano de Desarrollo. Recuperado de http://idbdocs.iadb.org/wsdocs/getdocument.aspx?docnum=1442235

Sautú, R. (1992). Teoría y medición del estatus ocupacional. Escalas ocupacionales objetivas y de prestigio. Buenos Aires: Facultad de Ciencias Sociales (UBA).

Semel, E., Wiig, E., \& Secord, W. (1995). Clinical Evaluation of Language Fundamentals Third Edition. San Antonio, TX: The Psychological Corporation.

Signorini, A., \& Borzone de Manrique, A. (2003). Aprendizaje de la lectura y escritura en español. El predominio de las estrategias fonológicas. Interdisciplinaria, 20, 5 - 30. Recuperado de https://www.redalyc.org/articulo.oa?id=18020102

Snow, C. (2018). Simple and Not-So-Simple Views of Reading. Remedial and Special Education, 39, 313-317.

Spencer, M., Quinn, J., \& Wagner, R. (2014). Specific Reading Comprehension Disability: Major Problem, Myth, or Misnomer? Learnging disabilities, Research and Practice, 29, 3-9.

Tobia, V., \& Bonifacci, P. (2015). The simple view of reading in a transparent orthography: the stronger role of oral comprehension. Reading \& Writing, 28, 939-957. https://doi.org/10.1007/s11145-015-9556-1

Tuñón, I. (2018). (In)equidades en el ejercicio de los derechos de niñas y niños. Buenos Aires: Barómetro de la Deuda Social de la Infancia, UCA. Recuperado de http://wadmin.uca.edu.ar/public/ckeditor/Observatorio\%20Deuda\%20Social/Document os/2018/2018-Observatorio-BDSI-Documento\%20Estad\%C3\%ADstico Infancia.pdf

Urquijo, S., Garcia Coni, A., \& Fernandes, D. (2015). Relación entre aprendizaje de la lectura y nivel socioeconómico en niños argentinos. Avances en Psicología Latinoamericana, 33(2) 303-318.

Valencia, R. (2010). Dismantling contemporary deficit thinking: Educational thought and practice. London: Routledge. 
Van den Broek, P. (1994). Comprehension and memory of narrative texts: Inferences and coherence. In M. A. Gernsbacher (Ed.), Handbook of psycholinguistics (pp. 539-588). San Diego, CA: Academic Press.

Van den Broek, P., Espin, C., McMaster, K., \& Helder, A. (2017). Developing reading comprehension interventions: Perspectives from theory and practice. En E. Segers, \& $P$. van den Broek (Ed.). Developmental Perspectives in Written Language and Literacy (pp. 85-101). Amsterdam: John Benjamins. https://doi.org/10.1075/z.206.06van

Wimmer, H., \& Schurz, M. (2010). Dyslexia in regular orthographies: Manifestation and causation. Dyslexia: an International Journal of Research and Practice, 16, 283-299. https://doi.org/10.1002/dys.411 